\title{
Battarrea stevenii (Liboschitz) Fr. EN PAPOSO II REGION DE CHILE
}

\section{(Battarrea stevenii (Liboschitz) Fr. in Paposo second Region of Chile)}

\author{
Hugo Madrid L. \\ Unitat de Microbiologia, Facultat de Medicina i Ciències de la Salut, \\ Universitat Rovira i Virgili, Sant Llorenç 21, 43201 Reus (Tarragona), España. \\ e-mail: hugo.madrid@urv.cat
}

Palabras clave: Basidiomycota, Battarrea stevenii, hongos gasteroides

Key words: Basidiomycota, Battarrea stevenii, gasteroid fungi

\section{RESUMEN}

Se reporta el hallazgo de Battarrea stevenii (Liboschitz) Fr., un raro basidiomiceto xerofítico, en la localidad de Paposo, II Región de Chile. Junto a la descripción e ilustración del material examinado, se entregan comentarios sobre su ecología, taxonomía y distribución.

\section{INTRODUCCION}

Durante el curso de trabajos de campo en ambientes xéricos del norte de Chile, se recolectó el carpóforo de un interesante basidiomiceto, el cual fue transportado al Museo Nacional de Historia Natural para su determinación. El estudio taxonómico permitió identificar este hongo como Battarrea stevenii (Libosch.) Fr. (Tulostomataceae), un raro taxon gasteroide reportado principalmente de zonas áridas, semiáridas y templadas (Moreno et al., 1986).

En el presente trabajo se describe e ilustra esta reciente colección chilena de $\boldsymbol{B}$. stevenii, junto a comentarios sobre su ecología, taxonomía y distribución.

\section{MATERIAL Y METODOS}

El material biológico fue colectado en la localidad de Paposo, II región, a unos $600 \mathrm{msnm}$. Es un sector de clima árido, influenciado por la neblina costera («camanchaca»). Entre la vegetación de la zona se

Recibido el 27 de Octubre 2007

Aceptado el 23 de Noviembre 2007

\section{ABSTRACT}

Battarrea stevenii (Liboschitz) Fr., a rare xerophytic basidiomycete is reported from Paposo, Second Region of Chile. Besides description and illustration of the examined samples, comments about its ecology, taxonomy and distribution are also discussed.

encuentran Alstroemeria violacea Phil., Chaetanthera glabrata (DC.) Meigen, Eulychnia breviflora Phil., Euphorbia lactiflua Phil., Lycopersicon chilense Dunal, Schizantus laetus Phil., y especies de Ephedra L. y Proustia Lag.

Para su observación al microscopio óptico, se realizaron preparaciones de la gleba en $\mathrm{KOH} \mathrm{3 \%} \mathrm{y}$ lactofenol.

Esta colección se conserva en el herbario de la Sección Botánica del Museo Nacional de Historia Natural de Chile.

\section{RESULTADOS Y DISCUSION}

Battarrea stevenii (Liboschitz) Fr., Systema Mycologicum 3: 7, 1829 (Figura 1).

Material estudiado: En suelo duro, cercano a la cactácea Eulychnia breviflora. Paposo, cerro el Mirador (24 59،, Lat. S; $70^{\circ} 26^{\prime}$ Long. O), II Región, Provincia de Antofagasta, Chile. 1-10 - 2005. Leg. Sergio Moreira. SGO 152678.

Macroscopía: fructificación estipitada. Saco esporífero de $36 \mathrm{~mm}$ de diámetro, en el que se observa una gleba 


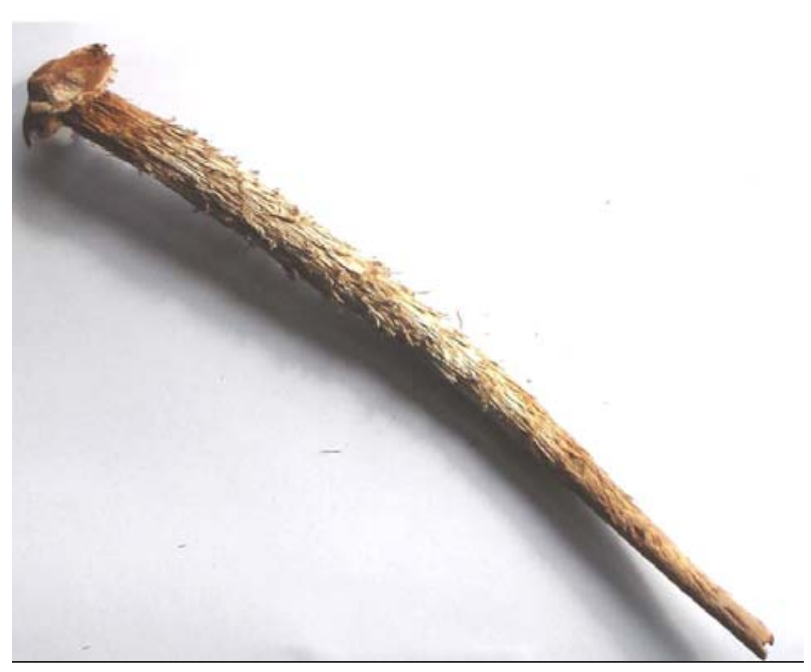

Figura 1. Basidiocarpo de Battarrea stevenii SGO 152678.

pulverulenta de color marrón rojizo sobre restos de un endoperidio blanquecino de consistencia coriácea. Estípite de 355 mm de longitud y 8-16 mm de diámetro, cilíndrico, adelgazado hacia la base, fistuloso, leñoso, blanquecino, cubierto por fibras y gruesas escamas que tienden a levantarse.

Microscopía: esporas globosas a subglobosas, de 5,2-6,4 x 5,5-6,4 $\mu \mathrm{m}$, verrucosas. Paracapilicio integrado por filamentos de 2,9-4 $\mu \mathrm{m}$ de ancho, lisos, hialinos, septados, en los cuales no se observan fíbulas. Eláteres de 31,349,3 $\mu \mathrm{m}$ de longitud y 4-7 $\mu \mathrm{m}$ de ancho, cilíndricos.

Observaciones: Battarrea habita zonas áridas, semiáridas y templadas del globo, con preferencia por ambientes con escasa precipitación pluvial (Moreno et al., 1986). Este género se ha reportado de África, América, Europa y Oceanía (Nieves-Rivera \& Lodge, 1998). En Chile, B. stevenii sólo se ha citado previamente de la IV Región, en el Parque Nacional Fray Jorge (Lazo, 1991). El hallazgo de este hongo en Paposo extiende el área conocida de su distribución en Chile.

El ejemplar de B. stevenii se observó creciendo solitario, en suelos duros, cercano a la cactácea $E$. breviflora. Aunque se han registrado asociaciones de Battarrea con especies de Acacia Mill., Atriplex L., Artemisia L., Eucalyptus L'Her., Euphorbia L., Juniperus L., Pinus L., Prosopis L., Sarcobatus Nees, Tamarix L. y Yucca L., no se ha demostrado la micorrización de dichas plantas, ni tampoco su posible parasitismo por el hongo. Al parecer, la principal fuente de nutrientes para Battarrea, sería la materia orgánica particulada presente en el suelo (Jacobson et al., 1999).
Las fructificaciones de Battarrea suelen comenzar a desarrollarse bajo el suelo. Inicialmente tienen una forma globoide o aplanada y están cubiertas completamente por el exoperidio, posteriormente, este se rompe, permitiendo distinguir tres partes en el carpóforo: una base, un estípite y una cabeza. Esta última contiene la gleba, rodeada por el endoperidio. La base permanece rodeada por una volva, compuesta por el exoperidio abierto y el mesoperidio. El estípite crece longitudinalmente, levantando el saco esporífero varios centímetros sobre la superficie del suelo, donde los propágulos quedan expuestos a corrientes de aire que permiten su diseminación. Para permitir la liberación de las esporas, el endoperidio se rompe, separándose por dehiscencia circuncisa en sus porciones superior e inferior. La porción superior puede perderse, a modo de un capuchón, dejando expuesta la gleba, que queda sostenida sobre la porción inferior del endoperidio, la cual permanece unida al estípite (Jacobson et al., 1999). La consistencia resistente y leñosa de los basidiomas permite que éstos perduren por largos períodos, una característica común en los basidiomicetos que habitan desiertos y otros ambientes sujetos a constante sequedad (Moreno et al., 1986).

El material examinado en el presente estudio corresponde a un ejemplar de $\boldsymbol{B}$. stevenii que ha perdido la porción superior del endoperidio, la volva y la mayor parte de la gleba. A nivel microscópico, es de importancia señalar que, en los filamentos del capilicio del material estudiado no se observaron fíbulas, las cuales sí se han mencionado en descripciones de material procedente de otras partes del mundo (Moreno et al., 1995; NievesRivera \& Lodge, 1998; Jacobson et al., 1999).

Otra especie, llamada Battarrea phalloides (Dickson) Pers., se diferenciaría de $\boldsymbol{B}$. stevenii principalmente por producir fructificaciones de menor tamaño, provistas de una volva gelatinosa (Cunningham, 1932). La separación de ambos taxa ha sido cuestionada por algunos autores, quienes han sugerido que podrían ser sinónimos (Rea, 1942; Moreno et al., 1995). Martín \& Johannesson (2000), compararon colecciones de $\boldsymbol{B}$. phalloides y B. stevenii procedentes de África, América y Europa. En dicho trabajo no se encontraron hifas gelatinizadas en la volva de $\boldsymbol{B}$. phalloides, ni ninguna otra característica morfológica que la distinguiera claramente de $\boldsymbol{B}$. stevenii. El análisis de secuencias nucleotídicas de las regiones ITS mostró un mayor número de diferencias entre distintas colecciones de $\boldsymbol{B}$. phalloides que entre esta especie y $\boldsymbol{B}$. stevenii. Estos datos respaldan la idea de que ambos taxa podrían ser sinónimos. Sin embargo, al no haberse incluido los holotipos en el estudio, la supuesta sinonimia entre B. phalloides y B. stevenii aún debe ser confirmada. 
El norte de Chile presenta características geográficas y climáticas aptas para el desarrollo de interesantes basidiomicetos xerofíticos (Philippi, 1860; Madrid \& Muñoz, 2006). Sin embargo, la información disponible sobre la micobiota del norte del país, es aún escasa y es evidente la necesidad de estudios adicionales.

\section{AGRADECIMIENTOS:}

A los profesionales de la Sección Botánica del Museo Nacional de Historia Natural de Chile. Al señor
Sergio Moreira y a la señora Mélica Muñoz, por haber facilitado el material estudiado e información sobre el sitio de recolección y la vegetación de la zona. A las señoras Elizabeth Barrera e Inés Meza, por la generosa acogida en sus laboratorios. A la señorita Laura Clavijo, por sus valiosos comentarios y colaboración técnica. A los expertos que han actuado como revisores de este manuscrito

\section{REFERENCIAS}

Cunningham, G. (1932). The Gasteromycetes of Australasia XIV. The family Tulostomataceae. Proc. Linn. Soc. New South Wales, 57:27-39

Jacobson, K.; Jacobson, P. \& Miller, O. (1999). The autoecology of Battarrea stevenii in ephemeral rivers of southwestern Africa. Mycol. Res. 103: 9-17

Lazo, W. (1991). Notas micológicas y bacteriológicas: Basidiomycetes y Streptomycetes de Chile. Bol. Micol. 6:41-42

Madrid, H. \& Muñoz, M. (2006). Primer registro de Montagnea arenaria (DC.) Zeller (Agaricales) en Chile. Rev. Iberoam. Micol. 23: 113-115

Martín, M. \& Johanneson, H. (2000). Battarrea phalloides and B. stevenii, insight into a long-standing taxonomic puzzle. Mycotaxon 74:67-75
Moreno, G.; Manjón, J. L. \& Zugaza, A. (1986). La Guía INCAFO de los hongos de la Península Ibérica. Vol. I y II. INCAFO. Madrid. España. Moreno, G., Altés, A., Ochoa, C., Wright, J. (1995). Contribution to the study of Tulostomataceae in Baja California, Mexico. I. Mycologia 87:96-120

Nieves-Rivera, A. \& Lodge, J. (1998). Contributions to the study of Gasteromycetes of Puerto Rico. Mc Ilvainea. 13:50-58

Philippi, R. (1860). Viage al desierto de Atacama, hecho de orden del gobierno de Chile en el verano 1853-54. Librería de Eduardo Anton, Halle, Alemania.

Rea, D. (1942). Fungi of Southern California I. Mycologia 34:563574 